\title{
Pilot study of sphenopalatine injection of onabotulinumtoxinA for the treatment of intractable chronic migraine
}

\author{
Daniel Fossum Bratbak ${ }^{1,2}$, Ståle Nordgård ${ }^{2,3}$, \\ Lars Jacob Stovner ${ }^{2,4}$, Mattias Linde ${ }^{2,4,5}$, David W Dodick ${ }^{2,6}$, \\ Irina Aschehoug ${ }^{2}$, Mari Folvik ${ }^{7}$ and Erling Tronvik ${ }^{2,4,5}$
}

\begin{abstract}
Objective: The main objective of this pilot study was to investigate the safety of administering onabotulinumtoxinA towards the sphenopalatine ganglion in 10 patients with intractable chronic migraine with an open, uncontrolled design. We also collected efficacy data to provide an indication as to whether future placebo-controlled studies should be performed.

Method: In a prospective, open-label, uncontrolled study after one-month baseline, we performed bilateral injections of $25 \mathrm{IU}$ onabotulinumtoxinA (total dose $50 \mathrm{IU}$ ) toward the sphenopalatine ganglion in a single outpatient session in 10 patients with intractable migraine with a follow-up of 12 weeks. The primary outcome was adverse events and the main efficacy outcome was frequency of moderate and severe headache days in month 2 post-treatment compared to baseline. Results: All 10 patients experienced a total of 25 adverse events. The majority of these were different types of local discomfort in the face and jaw, and none were classified as serious. In an intention-to-treat analysis of the main efficacy outcome, a statistically significant reduction of moderate and severe headache days in baseline versus month 2 was observed ( $16.3 \pm 6.2$ days baseline versus $7.6 \pm 7.6$ days month $2, p=0.009$ ). Eight out of 10 patients experienced an at least $50 \%$ reduction of moderate and severe headache days compared to baseline.

Conclusion: The result warrants randomised, placebo-controlled studies to establish both safety and efficacy of this potential novel treatment of chronic migraine.
\end{abstract}

\section{Keywords}

Chronic migraine, sphenopalatine ganglion, pterygopalatine ganglion, botulinum toxin, headache

Date received: I4 March 2016; revised: I8 March 2016; I April 2016; 6 April 2016; accepted: 7 April 2016

\section{Introduction}

Chronic migraine is a primary headache syndrome affecting approximately $1 \%-3 \%$ of the population, imposing a substantial burden on sufferers and society $(1,2)$. The use of prophylactic treatment in migraine is limited, probably due to lack of efficacy, side effects and contraindications $(3,4)$. Activation of parasympathetic nerves with their synapses in the sphenopalatine ganglion (SPG) is suggested to be an important mechanism in migraine (5), and has been targeted in several studies, mainly by various techniques of intranasal application of short-acting local anaesthetics for acute treatment, yielding mixed results (6). It has not convincingly been shown that these intranasally administered substances actually reach and hence are able to block the SPG.
'Department of Neurosurgery, St. Olavs Hospital, Trondheim University Hospital, Norway

${ }^{2}$ Department of Neuroscience, NTNU Norwegian University of Science and Technology, Norway

${ }^{3}$ Department of ENT, St. Olavs Hospital, Trondheim University Hospital, Norway

${ }^{4}$ Norwegian Advisory Unit on Headaches, St. Olavs Hospital, Trondheim University Hospital, Norway

${ }^{5}$ Department of Neurology, St. Olavs Hospital, Trondheim University Hospital, Norway

${ }^{6}$ Department of Neurology, Mayo Clinic, USA

${ }^{7}$ Department of Radiology, St. Olavs Hospital, Trondheim University

Hospital, Norway

\section{Corresponding author:}

Daniel Fossum Bratbak, Department of Neurosurgery, St. Olavs Hospital, Trondheim University Hospital and Department of Neuroscience, NTNU Norwegian University of Science and Technology, Olav Kyrres gate 17, 7006 Trondheim, Norway.

Email: daniel.f.bratbak@ntnu.no 
As a result, techniques have been developed to inject the pharmacological agents directly into the sphenopalatine fossa to overcome the possible limitations of the topical administration methods $(7,8)$.

In the SPG, preganglionic parasympathetic fibres synapse with postganglionic fibres innervating intracranial vessels using acetylcholine as the neurotransmitter. OnabotulinumtoxinA (BTA) causes neural block by inhibiting acetylcholine release, and in the autonomic system such blockage may last three to 12 months (9). Based on this knowledge, we have previously published an open-label study injecting BTA towards the SPG with a transnasal technique in intractable chronic cluster headache, showing significant improvement for the main efficacy parameter (10). A major limitation of the transnasal technique is that it requires general anaesthesia. In this study, we explored the potential of a long-lasting pharmacological blockade of the SPG in individuals with chronic migraine by a novel percutaneous approach enabling us to perform the treatment on awake patients under local anaesthesia in an outpatient office-based setting.

The main object of this pilot study was to investigate the safety of administering 25 IU BTA bilaterally (total dose $50 \mathrm{IU}$ ) towards the SPG in a single session in 10 patients with intractable chronic migraine using an open, uncontrolled design. We also collected efficacy data to determine whether future placebo-controlled studies should be performed.

\section{Method}

\section{Study design and participants}

The study was designed as a prospective, open-label, uncontrolled study and was conducted at St. Olavs Hospital, Trondheim, Norway, between December 2014 and February 2016. The study comprised a minimum four-week baseline period to record headache frequency, and a 12-week follow-up period. Ten patients with intractable chronic migraine were recruited either from the Neurology Department or by referrals from collaborating headache experts within Norway. Intractability was defined as patientreported unsatisfactory response or poor tolerability to at least three drugs shown to have effect in migraine. All patients recruited had been treated and evaluated by neurologist headache experts, ensuring that prior prophylactic medications had been used in adequate dose and duration. The exact limits of our definition are provided in the inclusion criteria. The treatment was performed with a novel surgical navigation device under clinical testing (MultiGuide - a safety study: Ref. regional ethics committee 2012/2199; ref. Directorate of Health 13/3816).
Inclusion criteria were age 18 to 70 years; chronic migraine according to the International Classification of Headache Disorders, third edition (ICHD-3 beta) (11); unsatisfactory effect, intolerable side effects or contraindications of at least three oral medications with documented effect in at least one placebo-controlled trial in episodic or chronic migraine from at least two of the following groups of medications: beta-blockers, calcium channel antagonist (flunarizine), angiotensin receptor blockers, antiepileptic drugs or antidepressants; duration of migraine at least one year before inclusion; onset of migraine before 50 years of age; and ability to differentiate migraine from other headaches.

Exclusion criteria were overuse of acute medication according to ICHD-3 beta (11); psychiatric illness preventing full participation; pregnancy, nursing or inability to use contraceptives in fertile women; abuse of any pharmacological substance, narcotics or alcohol; any disorder that could increase the risk of complications; hypersensitivity to short-acting anaesthetics, adrenalin or BTA; and active treatment with pharmacological substances with possible interaction with BTA.

The study treatment was assessed as an adjunctive therapy considered as appropriate for individuals with intractable chronic migraine by the International Headache Society's guidelines for chronic migraine trials (12), hence, patients on prophylactic treatment were not excluded if the effect was considered as unsatisfactory.

Qualified participants underwent physical and neurological examination. Medical history, computed tomography (CT) and magnetic resonance imaging (MRI) of the paranasal sinuses were obtained. Participants were instructed to keep headache diaries at least four weeks before treatment and throughout the follow-up recording headache frequency, duration (hours); intensity (graded mild, moderate, or severe); doses of acute treatment; days of sick leave; quality of life by Headache Impact Test-6 (HIT-6) (13) and adverse events (AEs).

Physician follow-up was conducted in week 4 and 12 after treatment in addition to weekly phone interviews by a study nurse for the first eight weeks. AEs were registered both by the physician and study nurse at follow-up using both open and specified questions.

The study was approved by the regional ethics committee (ref. 2014/962) and the Norwegian Medicines Agency (EUDRACT nr: 2014-001852-43) and is registered at ClinicalTrial.gov (NCT02259075). Written informed consent was obtained from all patients. Monitoring was performed by the Unit for Applied Clinical Research, NTNU Norwegian University of Science and Technology, Norway, to verify the appropriate adherence to protocol and the completeness of case reports form entries. 


\section{Procedure: BTA treatment of the SPG}

Since BTA is injected with a low volume compared to lidocaine, for example, and BTA has a low diffusion gradient (14), accuracy is of outmost importance to ensure that the BTA reaches the SPG. To comply with the accuracy needed, we developed a novel injection device to perform a surgical navigation-assisted administration of the drug (Figure 1).

A single treatment was performed on an awake participant in an outpatient office-based setting using a percutaneous, infrazygomatic approach with a novel injection device (MultiGuide ${ }^{\circledR}$, Trondheim, Norway; Figure 1) and aided by surgical navigation (Brainlab Kick version 1, Brainlab AG, Feldkirchen, Germany). Surgical navigation is a system that tracks and displays the tip of an instrument relative to a pre-acquired medical image. MultiGuide ${ }^{\circledR}$ enables the use of surgical navigation for high-precision injections on awake individuals. Pre-treatment planning of CT and MRI was performed with Brainlab iPlan 3.0 (Brainlab AG, Feldkirchen, Germany). The SPG was localised visually bilaterally and marked on fused MRI and CT scans. With the patient in a supine position, the skin and deep structures towards the sphenopalatine fossa were anaesthetised with 3-5 $\mathrm{ml}$ Marcaine-Adrenalin $(5 \mathrm{mg} / \mathrm{ml}+5 \mathrm{microgram} / \mathrm{ml}$, AstraZeneca Oslo, Norway) and a $1-2 \mathrm{~mm}$ skin incision was made. Aided by surgical navigation and MultiGuide ${ }^{\circledR}$, BTA was injected towards the SPG. Estimated duration of the injection is one to three minutes, and for the whole procedure including navigation system setup it is $20-30$ minutes. All patients received 25 IU BTA suspended in $0.5 \mathrm{ml}$ isotonic saline on each side for a total dose of $50 \mathrm{IU}$.

\section{Outcome and statistical analysis}

AEs (primary outcome) were registered and assessed from the treatment procedure to the end of the study. Secondary outcomes were the mean change from baseline to the second month of the treatment phase in frequency of days (defined as headache duration of $\geq 4$ hours or the use of triptans) with moderate or severe headache (main efficacy outcome); headache days with pain of any severity; migraine days; headache hours; migraine hours; headache severity index; headache intensity; days without headache; doses of analgesics; doses of triptans; acceptability of treatment; and headache-related impact on quality of life measured by the HIT-6. One month was defined as 28 days starting the day after treatment; migraine was defined as headache reported by patients in the headache diaries as migraine. For efficacy outcomes we have performed two types of analyses; intention-to-treat (ITT) analysis $(n=10)$ of all participants included and per-protocol (PP) analysis $(n=9)$ excluding protocol violators. A protocol violator was defined as a participant with less than $60 \%$ of diary days registered or change of prophylactic medication during the study. Missing values were estimated by using the last observation carried forward methodology. A responder analysis was performed for each month of the study period.

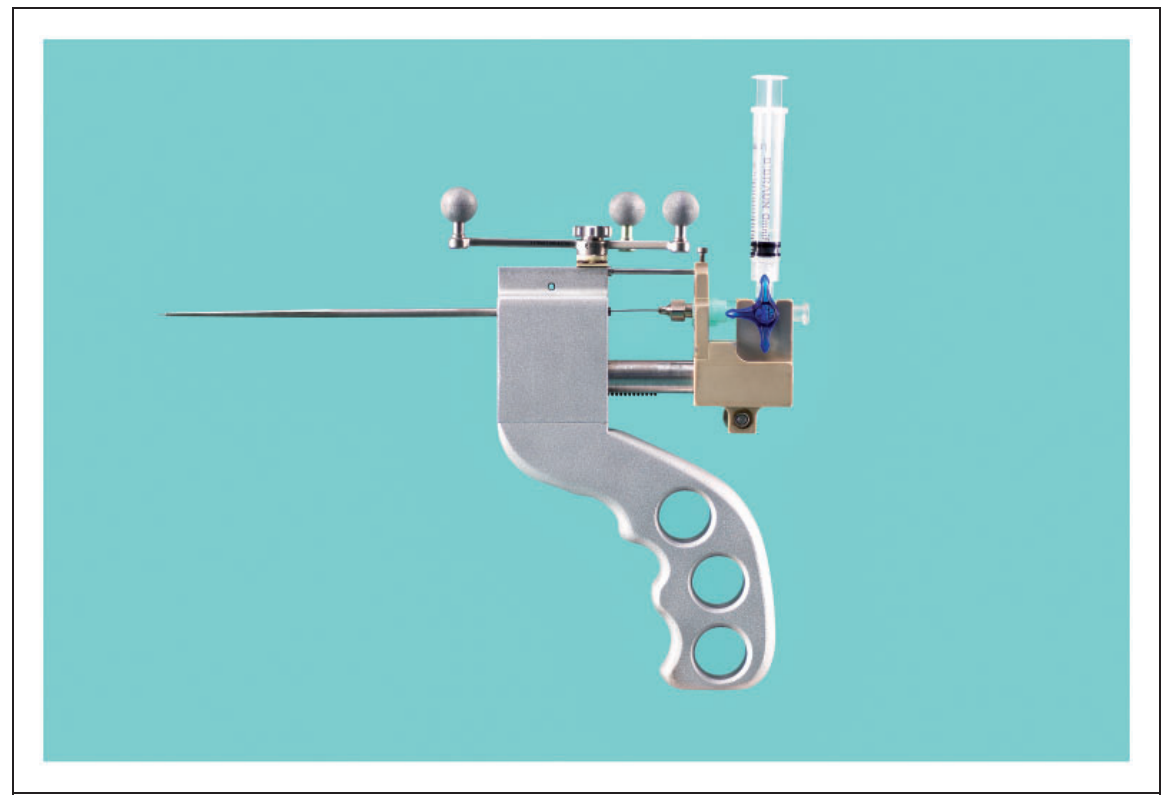

Figure I. Novel injection device to perform surgical navigation-assisted administration of drugs. 
A treatment responder (main efficacy outcome) was pre-defined as at least $50 \%$ reduction of moderate and severe headache days between baseline and month 2 .

SPSS version 21.0 (SPSS Inc, Chicago, IL, USA) was used in the data analyses. For efficacy measures we used the Wilcoxon signed rank test, and two-sided $p<0.05$ was considered statistically significant. Results are given as mean \pm standard deviation if not otherwise stated. Since the study is an exploratory safety study, no power calculation was performed prior to study start.

\section{Results}

Ten patients were included and all received the treatment. We received safety data on all patients throughout the study. Compliance with headache diary entry was $>90 \%$ for all participants. Seven of eight patients using migraine-prophylactic treatment were on stable doses at least two months prior to the intervention and throughout the study. Two patients did not use prophylactic medication during the study, and one patient discontinued prophylactic treatment the second month of the treatment study phase and was defined as a 'protocol violator' and excluded from the PP analysis. Demographics and clinical characteristics are given in Table 1.

\section{Safety (primary outcome)}

All 10 patients experienced AEs. Twenty-five events were registered, none classified as serious AEs (Table 2). All patients reported pain or other local symptoms related to the intervention, and five patients reported that these symptoms lasted for more than four weeks. Local symptoms were in general mild. One patient developed symptoms during month 2 compatible with temporomandibular joint dysfunction that persisted at the end of the study period. Clinical examination did not reveal sidespecific muscular weakness of the jaw; the relationship to the treatment is unknown. Investigators classified three treatment-emergent events as possibly related to the effect of BTA; one patient reported dry eye and three patients visual disturbances described as mild, intermittent visual blurring or accommodation problems.

\section{Efficacy outcomes}

Main efficacy outcome. For the main efficacy outcome, moderate and severe headache days in month 2, a statistically significant reduction from baseline was observed in the ITT analysis $(n=10), 16.3 \pm 6.2$ days
Table I. Demographics and clinical characteristics.

\begin{tabular}{|c|c|}
\hline & All patients $(n=10)$ \\
\hline Number of females/males & $10 / 0$ \\
\hline Mean age, years $\pm S D$ (range) & $44.7 \pm 14.5(24-68)$ \\
\hline $\begin{array}{l}\text { Mean years since onset of migraine } \pm S D \\
\quad \text { (range) }\end{array}$ & $28.8 \pm 11.7(15-49)$ \\
\hline $\begin{array}{l}\text { Mean years since onset of chronic } \\
\text { migraine } \pm S D \text { (range) }\end{array}$ & $16.8 \pm 1 \mid .4(4-40)$ \\
\hline $\begin{array}{l}\text { Number of evidence-based preventive } \\
\text { treatments failed }{ }^{\text {a }} \text {, mean } \pm \text { SD (range) }\end{array}$ & $4.3 \pm 1.5(3-7)$ \\
\hline $\begin{array}{l}\text { Number of all preventive treatments } \\
\text { failed }^{\mathrm{b}} \text {, mean } \pm \text { SD (range) }\end{array}$ & $6.8 \pm 3.1(4-11)$ \\
\hline Prophylactic medication use $\mathrm{e}^{\mathrm{a}, \mathrm{c}}$ & $8 / 10$ \\
\hline PREEMPT treatment failed ${ }^{d}$ & $10 / 10$ \\
\hline
\end{tabular}

a Level A to $C$ evidence (33) with unsatisfactory or intolerable side effects. ${ }^{b}$ All preventive treatments failed initiated by headache experts, including not evidence-based treatments.

'Stable doses of prophylactic medication use at inclusion.

${ }^{\mathrm{d}}$ At least one treatment failed; see Diener et al. (22) and Aurora et al. (23).

PREEMPT: Phase III Research Evaluating Migraine Prophylaxis Therapy.

baseline versus $7.6 \pm 7.6$ days month $2(p=0.009)$ (Figure 2). Number of moderate and severe headache days was decreased to $12.5 \pm 5.9(p=0.050)$ in month 1 , to $8.2 \pm 6.7(p=0.008)$ in month 3 (Figure 2), and for the whole follow-up period to $9.4 \pm 6.4(p=0.007)$. In the PP analysis $(n=9)$ moderate and severe headache days were reduced from $15.2 \pm 5.5$ in the baseline period to $5.3 \pm 2.8(p=0.008)$ in month 2 (Table 3$)$, and for the whole follow-up period to $7.5 \pm 1.9$ $(p=0.008)$.

Secondary efficacy outcomes. Summary statistics for secondary efficacy outcomes for the PP analysis are provided in Table 3. Statistically significant decreases from baseline were seen for almost all secondary variables. In the PP analysis the average reduction of moderate and severe headache days from baseline for the whole threemonth follow-up period was $51 \%(p=0.008)$. Eight patients were classified as treatment responders for the main efficacy outcome $(\geq 50 \%$ reduction of moderate and severe headache days between baseline and month 2, Table 4). Frequency changes for all months are provided in Table 4.

Headache impact on functioning and health-related quality of life. A statistically significant improvement of headache impact measured by the HIT- 6 was seen during the follow-up, with a mean decrease after four weeks of 9.2 points $(p=0.008)$, after eight weeks of 11.2 points $(p=0.008)$, and after 12 weeks of 13.8 points $(p=0.008)$ (Table 3$)$. 
Table 2. Reported adverse events (AEs), $n=10$.

\begin{tabular}{|c|c|c|c|}
\hline \multirow[t]{2}{*}{$A E$} & \multicolumn{3}{|c|}{ Number of patients } \\
\hline & $\begin{array}{l}\text { Resolved } \\
\leq 4 \text { weeks }\end{array}$ & $\begin{array}{l}\text { Resolved } \\
4-12 \text { weeks }\end{array}$ & $\begin{array}{l}\text { On-going at } \\
\text { end of study } \\
\text { period }\end{array}$ \\
\hline $\begin{array}{l}\text { Pain, swelling and numbness (face, cheek, temporomandibu- } \\
\text { lar joint, incision site, teeth, nose, palatine) }\end{array}$ & 5 & 3 & - \\
\hline $\begin{array}{l}\text { Jaw problems (reduced opening, muscle weakness, chewing } \\
\text { problems) }\end{array}$ & 5 & 2 & - \\
\hline Tearing & 1 & - & - \\
\hline Visual disturbances & - & 3 & - \\
\hline Mild dysphagia & 1 & - & - \\
\hline Nausea & 1 & - & - \\
\hline Dry eye & - & $\mathrm{I}$ & - \\
\hline Temporomandibular joint dysfunction & - & - & I \\
\hline Tinnitus & 1 & - & - \\
\hline Nasal obstruction & - & I & - \\
\hline Total (serious AE) & $14(0)$ & $10(0)$ & I (0) \\
\hline
\end{tabular}

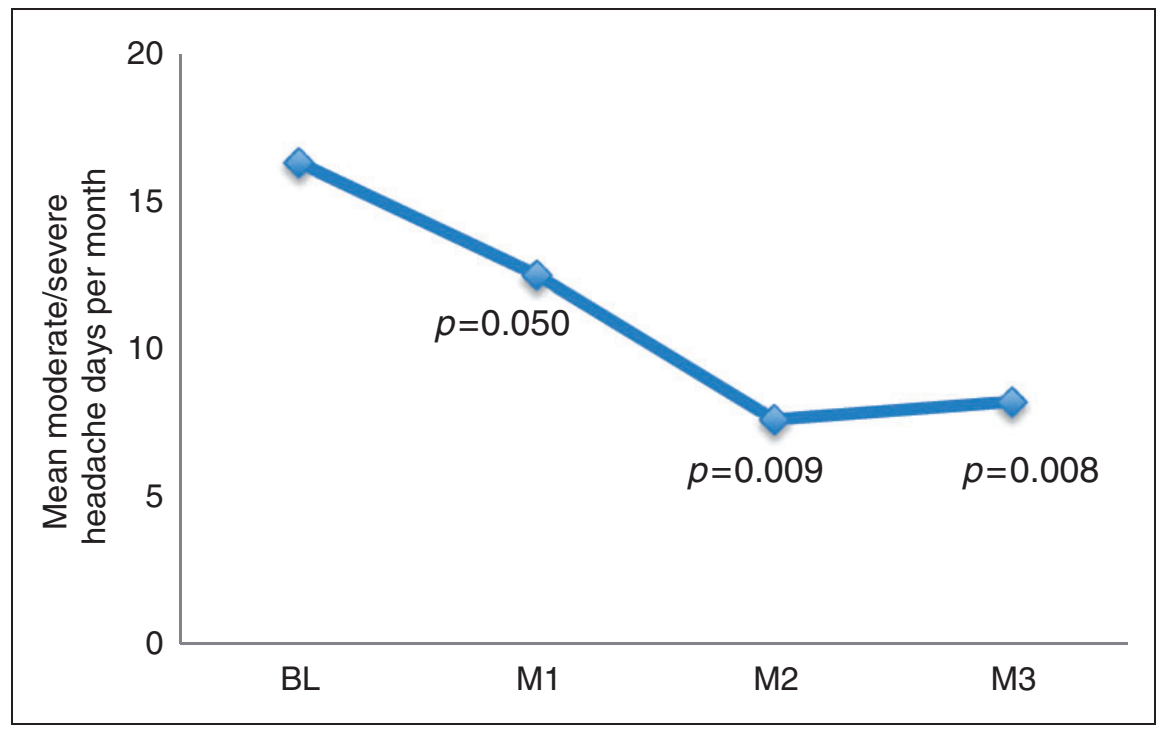

Figure 2. Main efficacy measure. Mean moderate and severe headache days per month for baseline and after one single, bilateral onabotulinumtoxinA treatment of the sphenopalatine ganglion, intention-to-treat analysis $(n=10)$.

BL: baseline; M: month (28 days).

$P$ value for each month compared to baseline.

Tolerability. On a visual analogue scale (1-10) the average maximal pain inflicted during the treatment was reported to be $2.1 \pm 1.5$, and one week after the treatment none were in need of analgesics due to treatment-related pain. At the end of the study period nine out of 10 patients would recommend the treatment to a friend, one would not due to lack of effect.
Nine out of 10 wanted to continue receiving treatments post-study.

\section{Discussion}

BTA injections towards the SPG have shown promising results in one open pilot study in patients with chronic 
Table 3. Secondary efficacy measures for baseline and after one single bilateral onabotulinumtoxinA treatment to sphenopalatine ganglion. Calculated by Wilcoxon signed rank test. Each month equals 28 days. Per protocol analysis, $n=9$.

\begin{tabular}{|c|c|c|c|c|}
\hline & $\begin{array}{l}\text { Baseline } \\
\text { Mean } \pm S D\end{array}$ & $\begin{array}{l}\text { Month I } \\
\text { Mean } \pm \text { SD } \\
(p \text { value })\end{array}$ & $\begin{array}{l}\text { Month } 2 \text { Mean } \pm S D \\
\text { ( } p \text { value })\end{array}$ & $\begin{array}{l}\text { Month } 3 \text { Mean } \pm S D \\
\text { ( } p \text { value) }\end{array}$ \\
\hline $\begin{array}{l}\text { Moderate or severe } \\
\text { headache days }^{\mathbf{a}}\end{array}$ & $15.2 \pm 5.5$ & $10.9 \pm 3.2(0.035)$ & $5.3 \pm 2.8(0.008)$ & $6.2 \pm 2.5(0.008)$ \\
\hline Headache days & $18.5 \pm 5.1$ & $14.6 \pm 4.3(0.038)$ & $7.6 \pm 1.4(0.008)$ & $7.3 \pm 2.3(0.008)$ \\
\hline Migraine days & $|4| \pm 4.8$. & $10.9 \pm 5.9(0.066)$ & $4.8 \pm 3.6(0.008)$ & $5.2 \pm 3.0(0.008)$ \\
\hline $\begin{array}{l}\text { Moderate or severe } \\
\text { headache hours }\end{array}$ & $137.2 \pm 85.3$ & $66.2 \pm 27.5(0.008)$ & $28.3 \pm 14.0(0.008)$ & $31.7 \pm 17.7$ (0.008) \\
\hline Migraine hours & || $8.5 \pm 78 . \mid$ & $70.4 \pm 53.8(0.01 \mathrm{I})$ & $23.2 \pm 18.0(0.008)$ & $26.1 \pm 19.2(0.008)$ \\
\hline Headache severity index ${ }^{b}$ & $60.8 \pm 61.2$ & $18.9 \pm 12.3(0.008)$ & $4.4 \pm 3.3(0.008)$ & $5.2 \pm 3.9(0.008)$ \\
\hline $\begin{array}{l}\% \text { Patients with severe } \\
(>60) \text { HIT-6 score }\end{array}$ & $100 \%$ & $67 \%$ & $56 \%$ & $22 \%$ \\
\hline HIT-6 score & $67.9 \pm 3.7$ & $58.7 \pm 6.0(0.008)$ & $56.7 \pm 7.3(0.008)$ & $54 . I \pm 7.3(0.008)$ \\
\hline Analgesics doses & $\mathrm{I} \mathrm{I} .5 \pm \mathrm{I} \mathrm{I} .7$ & $10.7 \pm 10.1(0.263)$ & $6.8 \pm 6.8(0.12)$ & $5.4 \pm 5.5(0.018)$ \\
\hline Triptan doses & $13.8 \pm 6.6$ & $1 \mathrm{II} .0 \pm 4.9(0.14)$ & $5.0 \pm 3.4(0.011)$ & $6.7 \pm 6.1(0.008)$ \\
\hline Days without headache & $9.2 \pm 5.4$ & $13.4 \pm 4.3(0.038)$ & $20.4 \pm 1.4(0.008)$ & $20.7 \pm 2.3(0.008)$ \\
\hline
\end{tabular}

$P$ value for each month compared to baseline.

${ }^{a}$ Categorical intensity scale; mild, moderate, severe.

bHours with moderate and severe headache per month multiplied with sum of intensity per month, $10^{-2}$.

cHIT: Headache Impact Test-6.

Table 4. Moderate and severe headache days per month (the main efficacy outcome) in each participants at baseline (BL) and percentage change each month $(\mathrm{M})$ post-treatment after one single bilateral onabotulinumtoxinA treatment to sphenopalatine ganglion. Each month equals 28 days.

\begin{tabular}{|c|c|c|c|c|c|c|}
\hline \multirow[b]{3}{*}{ Patient } & \multicolumn{6}{|c|}{ Moderate and severe headache days per month } \\
\hline & \multirow[b]{2}{*}{$\mathrm{BL}$} & \multicolumn{4}{|c|}{ Percentage reduction from $\mathrm{BL}$} & \multirow[t]{2}{*}{ Prophylactic medication use $\mathrm{a}^{\mathrm{a}}$} \\
\hline & & MI & M2 & M3 & $\mathrm{MI}$ to $\mathrm{M3}$ & \\
\hline I & 26 & $+4 \%$ & $+8 \%$ & $0 \%$ & $+4 \%$ & Amitriptyline $50 \mathrm{mg} /$ day \\
\hline 2 & 24 & $-33 \%$ & $-83 \%$ & $-67 \%$ & $-61 \%$ & Topiramate $225 \mathrm{mg} /$ day \\
\hline 3 & 10 & $+35 \%$ & $-14 \%$ & $-62 \%$ & $-14 \%$ & - \\
\hline 4 & 13 & $-40 \%$ & $-100 \%$ & $-70 \%$ & $-70 \%$ & $\begin{array}{l}\text { Candesartan } 16 \text { mg/day } \\
\text { Metoprolol LA } 100 \text { mg/day }\end{array}$ \\
\hline 5 & 15 & $-31 \%$ & $-52 \%$ & $-72 \%$ & $-52 \%$ & - \\
\hline 6 & 20 & $-40 \%$ & $-60 \%$ & $-\mathbf{5 0} \%$ & $-50 \%$ & Topiramate $50 \mathrm{mg} /$ day \\
\hline 7 & 15 & $-60 \%$ & $-53 \%$ & $-39 \%$ & $-51 \%$ & Candesartan $8 \mathrm{mg} /$ day \\
\hline 8 & 22 & $-49 \%$ & $-77 \%$ & $-86 \%$ & $-71 \%$ & Amitriptyline $50 \mathrm{mg} /$ day \\
\hline 9 & II & $+24 \%$ & $-52 \%$ & $-33 \%$ & $-21 \%$ & Candesartan $12 \mathrm{mg} /$ day \\
\hline 10 & 8 & $0 \%$ & $-63 \%$ & $-13 \%$ & $-25 \%$ & $\begin{array}{l}\text { Topiramate } 100 \mathrm{mg} / \text { day } \\
\text { Amitriptyline } 10 \mathrm{mg} / \text { day }\end{array}$ \\
\hline
\end{tabular}

Bold: Frequency responder a priori defined as $\geq 50 \%$ reduction of moderate and severe headache compared to baseline. M: month; BL: baseline; LA; long-acting.

${ }^{a}$ Level $A$ to $C$ evidence (33) with unsatisfactory or intolerable side effects.

b Stable doses of prophylactic medication use at inclusion. 
cluster headache using a transnasal technique performed under general anaesthesia (10). In the present study in 10 people with chronic migraine, the technique has been improved to enable us to perform BTA injections towards the SPG on awake patients under local anaesthesia in an outpatient office-based setting, making this potential treatment approach more practical for providers and more acceptable to patients. With this novel technique no serious AEs were reported. All patients experienced local symptoms after the injection, but in general these were perceived as mild. Local symptoms lasting longer than four weeks were reported by $50 \%$ of participants. Only one event, temporomandibular joint dysfunction first reported in the second month of the treatment phase and with unclear relation to the study treatment, was persistent at the end of the follow-up period. The AE profile seems acceptable given the level of disability associated with this disorder. The treatment was well tolerated and, at the end of the study period, nine out of 10 participants wanted to repeat the treatment when needed.

This is the first study to report results of BTA injections to the SPG in chronic migraine.

A unique feature of our study is the inclusion of severely intractable migraineurs (15) who had failed to respond to at least three evidence-based prophylactic treatments, and, in addition, acceptance for preventive drug use. In other studies, such as the Phase III Research Evaluating Migraine Prophylaxis Therapy (PREEMPT) trials (16), studies on topiramate (17-19) and calcitonin gene-related peptide (CGRP) monoclonal antibody studies (20), these patients are excluded. All our patients had failed on average 4.4 (range: 3-7) evidence-based, migraine-prophylactic drugs, and eight out of 10 patients were taking stable doses of prophylactic drugs at the time of study enrolment. Still, our results showed significant improvement of the main efficacy outcome in the ITT and PP analyses. In the PP analysis, the percentage reductions of moderate and severe headache for month 1, 2 and 3 were $28 \%, 65 \%$ and $59 \%$, respectively. Compared to pre-treatment baseline, all secondary efficacy outcomes showed statistically significant reductions at months 1 and 2 with the exception of triptan doses at month 1, migraine days at month 1 and analgesic use at months 1 and 2 . Even when targeting previously intractable patients, the magnitude of improvement indicated is clinically meaningful and in accordance with results reported for preventive therapies on less severely afflicted migraine populations (21).

Only BTA administered with the PREEMPT protocol is specifically approved for prophylaxis in chronic migraine. Two randomised, placebo-controlled studies with identical designs have investigated the PREEMPT method for prophylaxis in chronic migraine $(22,23)$. Two-thirds of the patient population overused acute medication and two-thirds had previously used headache-prophylaxis medication (16). In a pooled analysis of these two trials, mean reduction in headache days (the primary endpoint) in week 24 was 8.4 days/month in the BTA group and 6.6 days/month in the placebo group $(p<0.001)(16)$. The baseline headache frequency was 19.9 days/month, constituting a reduction of $42 \%$ in the active group and $33 \%$ in the placebo group. Common side effects in these studies included neck pain, other musculoskeletal pain, injection site pain, eyelid ptosis, muscular weakness and headache (21). All patients in our study were pharmacologically intractable and did not have satisfactory effect with at least one treatment of BTA according to the PREEMPT injection strategy. Despite that, headache days for the primary time point were reduced by $59 \%$ compared to baseline. While the PREEMPT studies are obviously of much higher quality and smaller studies are prone to overestimate effects, the proposed treatment, if proven effective and well tolerated in a randomised, placebo-controlled trial, may represent an alternative, especially in those not responsive to the PREEMPT protocol.

In animal studies, perivascular fibres of intracranial arteries have been traced back to the SPG $(24,25)$ and stimulation of the SPG induces dilatation of cranial blood vessels (26), plasma protein extravasation and release of inflammatory substances (27). Activation of parasympathetic fibres traversing the SPG may be involved in migraine pathophysiology $(28,29)$. In the SPG preganglionic parasympathetic fibres synapse with postganglionic fibres using acetylcholine as the neurotransmitter. BTA causes neural block by inhibiting acetylcholine release and may therefore block parasympathetic signalling through the SPG and hence inhibit perivascular release of neurotransmitters involved in migraine. Since sympathetic and sensory fibres do not synapse in the sphenopalatine fossa, it seems reasonable to posit that synaptic transmission will not be affected. No event of numbness or paraesthesia distant from the injection site was registered.

In the present study we performed one single treatment with effect lasting through the follow-up period, so duration of the treatment effect cannot be estimated. While autonomic block with BTA is known to last from three to 12 months (9), the duration of BTA injection to the SPG and the potential for additional therapeutic effect of repeated treatments should be investigated.

\section{Limitations}

Interpretation of efficacy data of this small, open study must be performed with great caution. For subjective 
parameters such as pain, the placebo response may be substantial. A meta-analysis of placebo response in prophylactic trials in migraine found a placebo response rate (reduction of migraine frequency by $\geq 50 \%$ ) for oral medications of $21 \%$ (30). Evidence suggests that parenteral treatments of pain have a higher placebo response than oral placebo (31). In the pooled data analysis of two randomised, placebo-controlled studies investigating the effect of 31 BTA injections to the pericranial and neck muscle in two cycles for prophylaxis in chronic migraine, a placebo response of $33 \%$ was reported (16). In addition to placebo, regression to the mean and periods of natural headache remission may bias the results in uncontrolled studies (32). However, while the sample is small, a responder rate of $80 \%$ in severely intractable patients who had suffered with chronic migraine for a mean of 16.8 years is notable. Overuse of acute medication was an exclusion criterion; we have not obtained information of prior medication overuse.

\section{Conclusion}

Our findings suggest that BTA injection to the SPG in patients with chronic migraine has an acceptable AE profile. The efficacy data indicate a statistically and clinically significant improvement for the main efficacy outcome, with eight out of 10 patients responding to the treatment. These results support the conduct of a randomised, placebo-controlled study to establish the safety, efficacy and duration of response of this possible novel treatment of chronic migraine.

\section{Clinical implications}

- In this pilot study our findings suggest that onabotulinumtoxinA (BTA) injection to the sphenopalatine ganglion (SPG) in chronic migraine has an acceptable adverse event profile.

- The efficacy data indicate a statistically and clinically significant improvement for moderate and severe headache (main efficacy outcome) and most secondary efficacy outcomes in severely intractable patients.

- Randomised, placebo-controlled studies are warranted to establish both safety and efficacy.

\section{Acknowledgements}

The authors would like to acknowledge the contribution of Dr Inge Monstad for his collaboration in this trial, and the Unit for Applied Clinical Research, NTNU Norwegian University of Science and Technology, Norway, for monitoring the study.

\section{Declaration of conflicting interests}

The authors declared the following potential conflicts of interest with respect to the research, authorship, and/or publication of this article: NTNU and St. Olavs Hospital, Trondheim University Hospital may benefit financially from a commercialisation of the proposed treatment through future possible intellectual properties; this may include financial benefits to authors of this article. Dr Bratbak is co-inventor of the proposed treatment in this study and the intervention device used to perform the treatment, both inventions patent pending, and may benefit financially from a commercialisation of the proposed treatment through future possible intellectual properties. Dr Nordgård is co-inventor of the proposed treatment in this study and the intervention device used to perform the treatment, both inventions patent pending, and may benefit financially from a commercialization of the proposed treatment through future possible intellectual properties. Dr Tronvik reports personal fees for one national advisory board meeting and reimbursement for scientific meetings from Allergan in 2014; and may benefit financially from a commercialisation of the proposed treatment through future possible intellectual properties. Dr Stovner reports personal fees from
GlaxoSmithKline outside the submitted work. Dr Linde has nothing to disclose. Dr Folvik has nothing to disclose. Irina Aschehoug has nothing to disclose. Dr Dodick has consulted for Allergan, Amgen, Alder, Boston Scientific, CoLucid, Merck, eNeura, Eli Lilly and Company, Autonomic Technologies, Teva, Tonix, Novartis, Supenus, Scion Neurostim, Xalan, Dr Reddy's and Xenon within the past 12 months.

\section{Funding}

The authors disclosed receipt of the following financial support for the research, authorship, and/or publication of this article: this work was supported by The Liaison Committee between the Central Norway Regional Health Authority and Norwegian University of Science and Technology (grant number 12/9996); Joint Research Unit between St. Olavs Hospital and Norwegian University of Science and Technology (grant number 9885); and NTNU Discovery (grant number 244278).

\section{References}

1. Stovner LJ and Andrée C. Impact of headache in Europe: A review for the Eurolight project. J Headache Pain 2008; 9: $139-146$.

2. Linde M, Gustavsson A, Stovner LJ, et al. The cost of headache disorders in Europe: The Eurolight project. Eur J Neurol 2012; 19: 703-711.

3. Lipton RB, Bigal ME, Diamond M, et al. Migraine prevalence, disease burden, and the need for preventive therapy. Neurology 2007; 68: 343-349. 
4. Hepp Z, Dodick DW, Varon SF, et al. Adherence to oral migraine-preventive medications among patients with chronic migraine. Cephalalgia 2015; 35: 478-488.

5. May A and Goadsby PJ. The trigeminovascular system in humans: Pathophysiologic implications for primary headache syndromes of the neural influences on the cerebral circulation. J Cereb Blood Flow Metab 1999; 19: 115-127.

6. Robbins MS, Robertson CE, Kaplan E, et al. The sphenopalatine ganglion: Anatomy, pathophysiology, and therapeutic targeting in headache. Headache 2016; 56: 240-258.

7. Prasanna A and Murthy PS. Sphenopalatine ganglion block under vision using rigid nasal sinuscope. Reg Anesth 1993; 18: 139-140.

8. Pipolo C, Bussone G, Leone M, et al. Sphenopalatine endoscopic ganglion block in cluster headache: A reevaluation of the procedure after 5 years. Neurol Sci 2010; 31(Suppl 1): S197-S199.

9. Naumann $\mathrm{M}$ and Lowe NJ. Botulinum toxin type $\mathrm{A}$ in treatment of bilateral primary axillary hyperhidrosis: Randomised, parallel group, double blind, placebo controlled trial. BMJ 2001; 323: 596-599.

10. Bratbak DF, Nordgård S, Stovner LJ, et al. Pilot study of sphenopalatine injection of onabotulinumtoxinA for the treatment of intractable chronic cluster headache. Cephalalgia 2016; 36: 503-509.

11. Headache Classification Committee of the International Headache Society (HIS). The International Classification of Headache Disorders, 3rd edition (beta version). Cephalalgia 2013; 33: 629-808.

12. Silberstein S, Tfelt-Hansen P, Dodick DW, et al. Guidelines for controlled trials of prophylactic treatment of chronic migraine in adults. Cephalalgia 2008; 28: 484-495.

13. Kosinski M, Bayliss MS, Bjorner JB, et al. A six-item short-form survey for measuring headache impact: The HIT-6. Qual Life Res 2003; 12: 963-974.

14. de Almeida AT and De Boulle K. Diffusion characteristics of botulinum neurotoxin products and their clinical significance in cosmetic applications. $J$ Cosmet Laser Ther 2007; 9(Suppl 1): 17-22.

15. Silberstein SD, Dodick DW and Pearlman S. Defining the pharmacologically intractable headache for clinical trials and clinical practice. Headache 2010; 50: 1499-1506.

16. Dodick DW, Turkel CC, DeGryse RE, et al. OnabotulinumtoxinA for treatment of chronic migraine: Pooled results from the double-blind, randomized, placebocontrolled phases of the PREEMPT clinical program. Headache 2010; 50: 921-936.

17. Silberstein SD, Lipton RB, Dodick DW, et al. Efficacy and safety of topiramate for the treatment of chronic migraine: A randomized, double-blind, placebo-controlled trial. Headache 2007; 47: 170-180.

18. Silvestrini M, Bartolini M, Coccia M, et al. Topiramate in the treatment of chronic migraine. Cephalalgia 2003; 23: $820-824$.
19. Diener HC, Bussone G, Van Oene JC, et al. Topiramate reduces headache days in chronic migraine: A randomized, double-blind, placebo-controlled study. Cephalalgia 2007; 27: 814-823.

20. Bigal ME, Edvinsson L, Rapoport AM, et al. Safety, tolerability, and efficacy of TEV-48125 for preventive treatment of chronic migraine: A multicentre, randomised, double-blind, placebo-controlled, phase $2 \mathrm{~b}$ study. Lancet Neurol 2015; 14: 1091-1100.

21. Schwedt TJ. Chronic migraine. BMJ 2014; 348: g1416.

22. Diener HC, Dodick DW, Aurora SK, et al. OnabotulinumtoxinA for treatment of chronic migraine: Results from the double-blind, randomized, placebo-controlled phase of the PREEMPT 2 trial. Cephalalgia 2010; 30: 804-814.

23. Aurora SK, Dodick DW, Turkel CC, et al. OnabotulinumtoxinA for treatment of chronic migraine: Results from the double-blind, randomized, placebo-controlled phase of the PREEMPT 1 trial. Cephalalgia 2010; 30: 793-803.

24. Suzuki N, Hardebo JE and Owman C. Origins and pathways of cerebrovascular vasoactive intestinal polypeptide-positive nerves in rat. $J$ Cereb Blood Flow Metab 1988; 8: 697-712.

25. Hardebo JE, Arbab M, Suzuki N, et al. Pathways of parasympathetic and sensory cerebrovascular nerves in monkeys. Stroke 1991; 22: 331-342.

26. Goadsby PJ. Sphenopalatine ganglion stimulation increases regional cerebral blood flow independent of glucose utilization in the cat. Brain Res 1990; 506: 145-148.

27. Delépine L and Aubineau P. Plasma protein extravasation induced in the rat dura mater by stimulation of the parasympathetic sphenopalatine ganglion. Exp Neurol 1997; 147: 389-400.

28. Yarnitsky D, Goor-Aryeh I, Bajwa ZH, et al. 2003 Wolff Award: Possible parasympathetic contributions to peripheral and central sensitization during migraine. Headache 2003; 43: 704-714.

29. Olesen J. The role of nitric oxide (NO) in migraine, tension-type headache and cluster headache. Pharmacol Ther 2008; 120: 157-171.

30. Macedo A, Baños JE and Farré M. Placebo response in the prophylaxis of migraine: A meta-analysis. Eur J Pain 2008; 12: 68-75.

31. Kaptchuk TJ, Stason WB, Davis RB, et al. Sham device v inert pill: Randomised controlled trial of two placebo treatments. BMJ 2006; 332: 391-397.

32. Diener HC, Schorn CF, Bingel U, et al. The importance of placebo in headache research. Cephalalgia 2008; 28 : 1003-1011.

33. Estemalik E and Tepper S. Preventive treatment in migraine and the new US guidelines. Neuropsychiatr Dis Treat 2013; 9: 709-720. 